\title{
Aneutronic pB-Reaction in Magnetized Inertial Confined Plasma of Laser-Accelerated Particles
}

\author{
Sergey Gus'kov ${ }^{1,2 *}$ Philipp Korneev ${ }^{1,2}$ \\ ${ }^{1}$ LPI RAS, 119991, Moscow Leninskii pr. 53, Russian Federation \\ ${ }^{2}$ NRNU MEPhI, 115409 Moscow, Kashirskoe sh. 31, Russian Federation
}

\begin{abstract}
A brief review on studies aimed at initiating a neutronless nuclear reaction between protons and boron nuclei is presented. The least energy-cost of a currently known approach to ignition of $p B$ reaction is proposed using a petawatt laser for producing a magnetized plasma of laser-accelerated ions and electrons as well as using a terawatt laser for inertial confinement of such a plasma.
\end{abstract}

\section{Introduction}

Promising aneutronic nuclear power based on the reaction of a proton with boron, which gives three $\alpha$ particles with a total energy of about $\varepsilon \approx 8.6 \mathrm{MeV}$, is currently under active discussion. As compared to other aneutronic reactions, $p B$ reaction has the largest cross section in the region of the main resonance, $\sigma_{\max } \approx 1 \mathrm{~b}$ (at the energy of the relative motion of the nuclei $E_{\text {rel }} \approx 675$ $\mathrm{keV}$ ), which is nevertheless much smaller than the maximum cross section for the reaction of deuterium with tritium ( $6 \mathrm{~b}$ at $\left.E_{\mathrm{rel}} \approx 60 \mathrm{keV}\right)$. The initiation of the $p B$ reaction in the plasma is experimentally studied with the use of laser methods of creation of a hightemperature plasma and a proton beam [1-4]. The maximum detected yield of $\alpha$ particles in the $p B$ reaction was about $10^{9}$ [3]. Due to a rather small cross section, the initiation of an energetically profitable $p B$ reaction requires a significant initial energy expense much higher as compared to the fusion reaction between hydrogen isotopes. Nevertheless, several approaches were proposed to solve this problem. They are (i) the use of an external magnetic field for the quasi stationary interaction between the colliding proton beam and boron nuclei [5], (ii) initiation of the reaction in a degenerate plasma with a superhigh density of about $10^{5} \mathrm{~g} / \mathrm{cm}^{3}$ [6], (iii) action of laser-accelerated proton beams on a preliminarily compressed boron-containing target [7], and (iv) the collision between ponderomotively accelerated thin layers of matter [8]. All these approaches are devoted to reduce the effect of Coulomb breaking of protons and boron nuclei initially accelerated to $\mathrm{MeV}$ energies.

Here we discuss the least energy-cost of currently known approach to ignition of $p B$ reaction, which has been proposed in [9]. It is based on the inertial confinement of a magnetized plasma of laser-accelerated charged particles. The features of this approach are, first, the creation of the magnetized $p B$ plasma in the inner cavity of the target in the form of a spherical or

*Corresponding author: guskov@sci.lebedev.ru cylindrical shell owing to the irradiation of its inner surface by a picosecond petawatt laser pulse and, second, the compression and confinement of such a plasma owing to the irradiation of the outer surface of the target by a nanosecond terawatt laser pulse. In order to match the processes of formation and compression of the plasma, a plasma producing pulse should be applied at the final stage of acceleration of the target under the action of a compressive pulse. It is proposed to use targets similar to the spherical $[10,11]$ or cylindrical [12] target designed for fast ignition [10, 13] of the preliminarily compressed plasma at the inertial confinement (see Fig. 1). In both cases, compression occurs in the radial direction. The spherical target is compressed along the conical guiding channel and the cylindrical target with the shape of a coil is compressed along the end walls. Holes in the vertex of the conical channel and end walls serve for the introduction of the plasma-producing laser pulse.
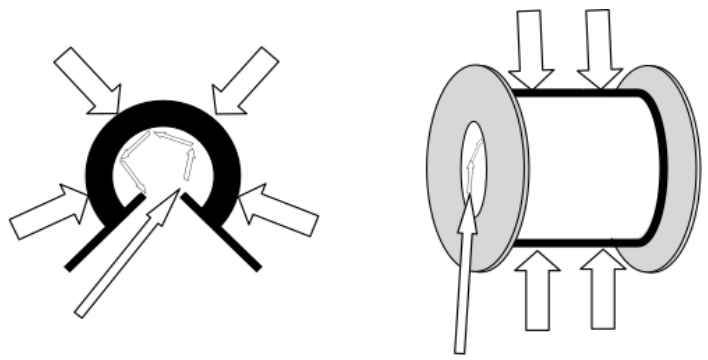

Fig. 1. Spherical (left) and cylindrical (right) targets for the compression and inertial confinement of the magnetized plasma formed at the grazing incidence of a relativistic laser pulse on the surface of the inner cavity of the target. The thin arrows correspond to the beams of petawatt laser, the thick ones corresponds to the beams of terawatt laser.

The creation of the magnetized plasma of laser accelerated ions and electrons was predicted in [14] on the basis of the PIC simulation of the interaction of a relativistic laser pulse with an intensity of $10^{19} \mathrm{~W} / \mathrm{cm}^{2}$ 
with the inner surface of a quasicylindrical target. Radiation tangentially incident on the curved surface of the target generates surface currents of fast electrons. They generate a flow of fast ions and discharge currents of thermal electrons. In the region between the two currents, a high magnetic field is generated and is carried together with the plasma of laser-accelerated and thermal particles into the cavity of the target; i.e., the ablation of the magnetized plasma inside the target occurs. The numerical calculations in [14] indicate the formation of a plasma with $\mathrm{MeV}$ particles, where the density exceeds the critical plasma density $\left(10^{21} \mathrm{~cm}^{-3}\right)$. The time of existence of the magnetic field with a strength of several hundred megagauss was much longer than the duration of the pulse. The kinetic energy of the shell at the time of the end of the creation of the plasma should be enough to compress the plasma to a density necessary for breakeven, i.e., for the release of the energy in the $p B$ reactions equal to the energy of the plasma. This requirement determines the relations between the parameters of the plasma-producing and compressive pulses.

\section{Magnetized plasma of laser- accelerated Mev ions}

To numerically simulate the formation of the $p B$ plasma a model substance as a mixture of three parts of hydrogen and one part of boron was used in PIC simulations. The shape of the target was specified by the expression $r(\theta)=r_{0}\left(1+\theta \delta r / 2 \pi r_{0}\right), \theta \subset(0,2 \pi)$. The angle $\theta=0$ corresponds to the upward direction along the $y$ axis in Fig. 2 and the parameters were $\delta r=50 \mu \mathrm{m}$ and $r_{0}$ $=98 \mu \mathrm{m}$. The two-dimensional geometry, limited computation region, and limited duration of calculation (9.5 ps) were taken according to the available computational capabilities. A helical shape of the target was used for the two-dimensional simulation of the penetration of a laser pulse into the cavity of the target after its tangential incidence and multiple reflections from the walls of the target, which is the most important phenomena here.

The laser pulse had an intensity of $10^{19} \mathrm{~W} / \mathrm{cm}^{2}$ and a duration of $1.7 \mathrm{ps}$, the wavelength of radiation was 1.06 $\mu \mathrm{m}$, and the radius of the beam was $20 \mu \mathrm{m}$. The electron density $n_{e}=4.5 \cdot 10^{22} \mathrm{~cm}^{-3}$ corresponded to 45 critical densities. The spatial and time steps were $20 \mathrm{~nm}$ and $0.07 \mathrm{fs}$, respectively. Figure 2 shows the spatial distributions of electron fluxes and the strength of the magnetic field at the time of the end of the laser pulse $t=$ 1.7 ps. The initial shape of the target is also presented and the trajectory of the laser pulse, as well as the region filled with the plasma, are qualitatively shown.

The characteristic plasma density in the region of interaction of the laser beam with the plasma near the boundary of the cavity was $4 \cdot 10^{21} \mathrm{~cm}^{-3}$. A more detailed description of the spatial distributions of the parameters of the plasma and magnetic field, as well as of their time evolution, was given in [9]. Figure 3 shows the energy distributions of electrons at $t=1.7$ and $9.5 \mathrm{ps}$, as well as of protons and boron nuclei at $t=9.5 \mathrm{ps}$ in the inner region of the target. The energy of protons and boron nuclei in the central region of the target is several $\mathrm{MeV}$, and the strength of the field, which is "frozen" in the plasma at such a high energy of particles, is about 100 MG. In such a field, the Larmor radii of boron nuclei, protons, and $3-\mathrm{MeV} \alpha$ particles from the $p B$ reaction are about 10,3 , and $50 \mu \mathrm{m}$, respectively.

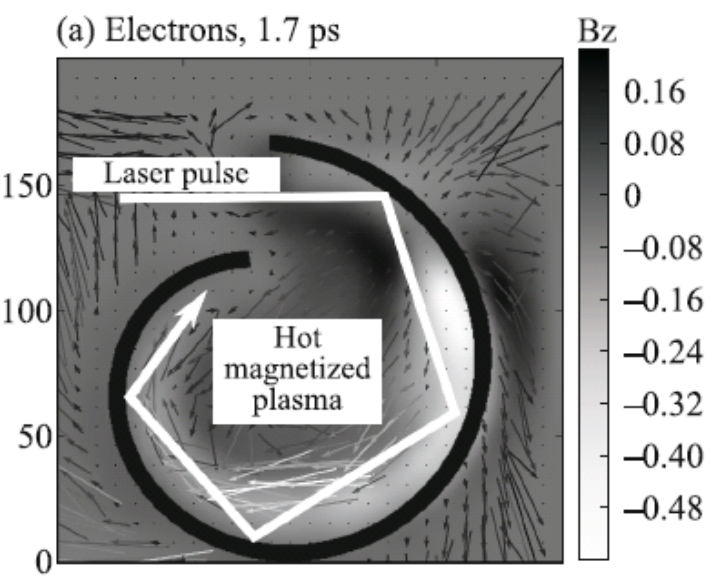

Fig. 2. Spatial distribution of the electron flows and magnetic field at a time of $1.7 \mathrm{ps}$. The momenta of the electrons are demonstrated by arrows in units of $m_{e} c$, the strengths of the magnetic field $B z$ in the direction perpendicular to the figure plane are shown as the background of the figure according to the color scale at the same times.

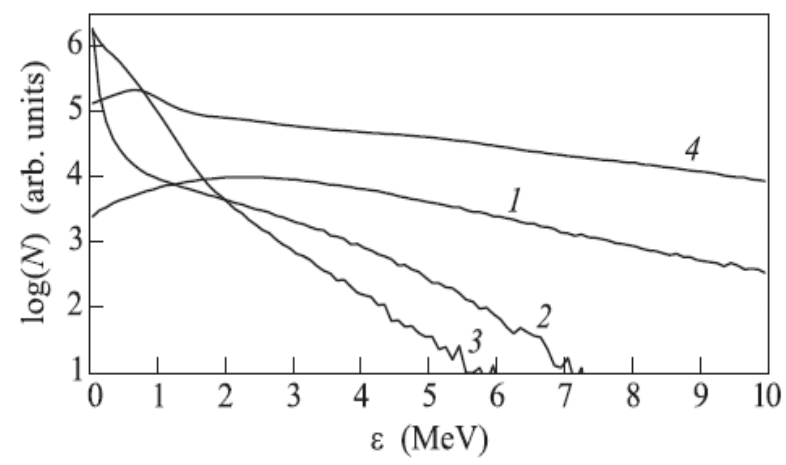

Fig. 3. Energy spectra of electrons at a time of 1.7 ps (line 1) and 9.5 ps (line 2) as well as of protons (line 3 ) and boron nuclei (line 4) at a time 9.5 ps in the inner region of the target inside a distance of $50 \mu \mathrm{m}$ from the center of the target.

\section{Lawson's criterion for pB-plasma}

In view of the possibility of the producing of the magnetized plasma with $\mathrm{MeV}$ light nuclei in the inner cavity of the target, we estimate the conditions for the initiation of the $p B$ reaction in the following formulation of the problem. A plasma-producing laser pulse with the intensity $I_{1}$, energy $E_{1}$, and duration $\tau_{1}$ is guided through a transport channel to the inner surface of the spherical $p B$ shell in the final stage of its acceleration under the action of a laser pulse with the respective parameters $I_{2}$, $E_{2}$, and $\tau_{2}\left(\tau_{2}>>\tau_{1}\right)$. The velocity of flight of the shell to the center $u$ and the radius of its inner surface $R_{0}$ at the beginning of the action of the plasma-forming pulse correspond to the condition under which the time of the 
convergence of the shell to the center $t_{h}=R_{0} / u$ is much larger than the duration of the plasma-producing pulse: $t_{h}>>\tau_{1}$. Although the frequencies of collisions involving laser-accelerated particles are very low, as will be shown below, the electron-ion and, the more so, ion-ion energy relaxation processes occur in the time of the compression of the plasma. Then, under the assumptions that (i) the average energy of particles of the plasma formed at the interaction between flows of laser-accelerated and thermal particles is $\varepsilon \approx 500 \mathrm{keV}$ (corresponds to the kinetic energy of $675 \mathrm{keV}$ of nuclei in the center-of-mass system), (ii) the number of protons is equal to the number of boron nuclei, and (iii) the plasma is quasineutral, the ratio of the energy released in nuclear reactions to the energy of the plasma is $G \approx \varepsilon_{r} n_{c} \sigma_{\max } v t_{r} / 8 \varepsilon$ $\approx 2.1 \cdot 10^{-15} n_{c} t_{r}$, where $v=\left(2 \varepsilon / m_{p}\right)^{1 / 2}$ is the relative velocity of nuclei, which is approximately equal to the velocity of protons; $m_{p}$ is the mass of the proton; $n_{c}$ is the density of nuclei in the plasma in $\mathrm{cm}^{-3}$; and $t_{r}$ is the time of the confinement of the plasma in seconds. Consequently, the condition of breakeven, when the requirement $G>1$ (Lawson' criterion) is satisfied for the $p B$ reaction has the form

$$
n_{c r} t_{r}>4.5 \cdot 10^{14} \mathrm{~s} / \mathrm{cm}^{3}
$$

\section{Compression and inertial confinement of magnetized pB-plasma of laser accelerated particles}

Now the requirements to the degree of compression and the duration of confinement of magnetized $500-\mathrm{MeV}$ pB-plasma under the action of terawatt laser pulse will be determined which are necessary to achieve Lawson's criterion (1). The degree of compression of the plasma can be estimated in the adiabatic approximation from the condition of the transfer of the kinetic energy of the shell to the internal energy of the plasma decelerating it

$$
\frac{n_{c}}{n_{0}} \equiv\left(\frac{R_{0}}{R_{c}}\right)^{3} \approx\left(\frac{\eta_{2} E_{2}}{\eta_{1} E_{1}}\right)^{1 /(\gamma-1)}
$$

where

$$
n_{0}=\frac{3 \eta_{1} E_{1}}{32 \pi R_{0}^{3} \varepsilon} \approx 3.7 \cdot 10^{11} \frac{\eta_{1} E_{1}}{R_{0}^{3}}
$$

is the density of nuclei in the plasma produced by the petawatt pulse; $n_{c}$ and $R_{c}$ are the density of the compressed plasma and corresponding radius of the shell, respectively; $\eta_{1}$ is the fraction of the energy of the plasma-producing laser pulse spent on the formation of the plasma; $\eta_{2}$ is the hydrodynamic efficiency of energy transfer [15], which is the ratio of the kinetic energy of the shell to the energy of the compressive laser pulse; and $E_{1}$ and $R_{0}$ are measured in joules and seconds, respectively. In view of Lawson's criterion (1), the time of confinement of the plasma in the shell with the mass $M_{c}$ is given by the expression

$$
t_{r} \approx R_{c}\left[\frac{(\gamma-1) \eta_{1} E_{1}}{M_{c}}\right]^{-1 / 2} \approx 1.73 \frac{R_{0}}{u}
$$

The substitution of Eqs. (2)-(4) into Eq. (1) gives

$$
\frac{\eta_{1} E_{1}}{R_{0}^{2} u}\left(\frac{\eta_{2} E_{2}}{\eta_{1} E_{1}}\right)^{3 / 2}>7 \cdot 10^{2}
$$

where $u$ is measured in centimeters per second.

For further analysis, we introduce a number of additional constraints. To simplify the focusing of the plasma-producing beam with the radius $R_{1}$ on the inner surface of the shell at the final stage of its acceleration, we set $R_{0} / R_{1}=5$. Below, for the stability of compression, we take the relatively small ratio $R_{0} / R_{c}=5$, which corresponds to the degree of compression $n_{c} / n_{0}=125$. In this case, according to Eq. (2) at $\gamma=5 / 3, \eta_{2} E_{2} / \eta_{1} E_{1}=25$. Taking into account the accepted relations, we estimate the initial radius of the cavity as $R_{0} \approx 1.8 \cdot 10^{3} u^{2} / \eta_{1} I_{1}$ according to Eq. (5). Taking $\eta_{1}=1$, the velocity $u=300$ $\mathrm{km} / \mathrm{s}$ as a typical value for inertial confinement fusion, and the intensity $I_{1}=10^{20} \mathrm{~W} / \mathrm{cm}^{2}$, we estimated the radius of the cavity as $R_{0} \approx 180 \mu \mathrm{m}$, the time of compression as $t_{h} \approx 600 \mathrm{ps}$, and the other parameters of the plasma-forming pulse as $R_{1}=36 \mu \mathrm{m}, \tau_{1} \approx 15 \mathrm{ps}$, and $E_{1}=50 \mathrm{~kJ}$. According to Eqs. (3) and (2), the initial and final densities of nuclei in the $p B$ plasma are, respectively, $n_{0} \approx 3.4 \cdot 10^{21} \mathrm{~cm}^{-3}$ and $n_{c} \approx 4.2 \cdot 10^{23} \mathrm{~cm}^{-3}$ (larger than the density of solid boron by a factor 3 ) and the mass of the plasma is $m \approx 1.6 \mu \mathrm{g}$. The calculation shows that the ion-ion energy relaxation time (about 2 ps) is much shorter than the plasma compression time, whereas the electron-ion energy relaxation time (about 1 ns) is close to this time.

Energy losses to cyclotron radiation in a time of about $1 \mathrm{~ns}$ in a field of $100 \mathrm{MG}$ are no more than a few percent. The above analysis was performed in the approximation of full magnetization of the electron heat conduction to the shell compressing the plasma. For this reason, the field does not appear in the expression for the ignition criterion. A more accurate calculation should certainly include the dependence on the field caused by the field dependence of energy losses owing to the electron heat conduction. The parameters of the laser pulse required for the compression of a spherical inertial confinement fusion target were chosen such that approximately $80 \%$ of the mass of the shell is evaporated in the process of acceleration. In this case, the hydrodynamic efficiency is close to its maximum possible value of about $30 \%$. Then, with the use of the parameters $\eta_{2}=0.3, \eta_{2} E_{2} / E_{1}=25$, and $E_{1}=50 \mathrm{~kJ}$, the energy of the compressive pulse should be $E_{2}=4.1 \mathrm{MJ}$. The final mass and velocity of the spherical shell at laser ablation are (see, e.g., [15]) 


$$
\begin{gathered}
u_{c} \approx 2 u_{a v}=2\left(\frac{2}{3}\right)^{1 / 3} \alpha^{1 / 2} V_{s} \\
M_{c}=M_{0}\left(1-\frac{2}{3} \alpha^{1 / 2}\right)
\end{gathered}
$$

respectively. Here, $u_{\mathrm{av}}$ is the average velocity of the shell, $V_{\mathrm{s}}=\left(I_{2} / 3 \rho_{\mathrm{cr}}\right)^{1 / 3}$ is the speed of sound in the fully ionized plasma of the evaporated part of the target, and $\alpha$ $=\rho_{\mathrm{cr}} R_{\mathrm{s} /} \rho_{\mathrm{s}} \Delta_{\mathrm{s}}$ is the dimensionless acceleration parameter, where $R_{\mathrm{s}}, \Delta_{\mathrm{s}}$, and $\rho_{\mathrm{s}}$ are the initial radius, thickness, and density of the material of the shell, in microns, respectively; and $\rho_{\mathrm{cr}} \approx 3.6 \cdot 10^{-3} \lambda_{\mu \mathrm{m}}{ }^{-2}$ is the critical plasma density, where $\lambda_{\mu \mathrm{m}}$ is the wavelength of radiation in microns.

For the pulse of the third harmonic of the Nd laser for which the critical density is $0.032 \mathrm{~g} / \mathrm{cm}^{3}$ and for the target with $\rho_{0} \approx 2.34 \mathrm{~g} / \mathrm{cm}^{3}$ at $M_{c} / M_{0}=0.2$ and $u_{c}=3 \cdot 10^{7}$ $\mathrm{cm} / \mathrm{s}$, estimates by Eqs. (6) and (7) give $\alpha \approx 1, R_{\mathrm{s}} / \Delta_{\mathrm{s}} \approx$ 70 , and $I_{2} \approx 5 \cdot 10^{13} \mathrm{~W} / \mathrm{cm}^{2}$. This value of the intensity $I_{2}$ at the duration of laser pulse equal to the time of shell implosion $\tau_{2}=2 R_{\mathrm{S}} / u_{c}$ corresponds to the radius of the target $R_{\mathrm{s}}=0.46 \mathrm{~cm}$. At the aspect ratio $R_{\mathrm{s}} / \Delta_{\mathrm{s}} \approx 70$, this radius corresponds to the thickness of the shell of $65 \mu \mathrm{m}$, the final mass of the shell $M_{c} \approx 8 \mathrm{mg}$, and the duration of the pulse $\tau_{2}=30 \mathrm{~ns}$. Summarizing the parameters of the compressive pulse of the third harmonic of the $\mathrm{Nd}$ laser are $I_{2} \approx 5 \cdot 10^{13} \mathrm{~W} / \mathrm{cm}^{2}, E_{2}=4.1 \mathrm{MJ}$, and $\tau_{2}=30 \mathrm{~ns}$. The total energy $E_{2}+E_{1}=4.15 \mathrm{MJ}$ is more than five times higher than the energy of the laser pulse necessary for the compression and ignition of the traditional inertial confinement fusion target with the DT fuel (see, e.g., [16]). Such a high energy is due primarily to a significantly higher energy of the shell required for the compression of the $p B$ plasma preliminarily heated by a relativistic pulse.

The ratio of the final mass of the shell to the mass of the compressed plasma $M_{c} / m$ is about $5 \cdot 10^{3}$. The total gain $G_{\mathrm{t}}$ equal to the ratio of the energy released in $p \mathrm{~B}$ reactions to the total spent energy $E_{1}+E_{2} \approx E_{2}$ at the propagation of combustion to the compressed material of the shell can be larger than $G_{\mathrm{t}} \approx M_{c} E_{1} / m E_{2} \approx 60$, which is sufficient for the positive energy cycle including the efficiency of the laser. However, the initiation of a combustion wave with allowance for the features of the problem under consideration requires separate analysis.

\section{Conclusion}

The presented justification of the fundamental possibility of the least energy-cost of currently known approach to ignition of $p B$ ignition of the magnetized $p B$ plasma produced in the inner cavity of the compressed inertial confinement fusion target implies a further study of the dependences of the parameters of the $p B$ plasma, the strength of the spontaneous magnetic field, and the time of conservation of the magnetized plasma state on the parameters of the plasma-producing pulse.
This work was supported by the Russian Foundation for Basic Research (project no. 16-52-50019 YaF).

\section{References}

1. V. S. Belyaev, A. P. Matafonov, V. I. Vinogradov, V. Krainov, P. Lisitsa, V. S. Roussetski, A. S. Ignatyev, and G. N. Andrianov, Phys. Rev. E 72, 026406 (2005)

2. C. Labaune, S. Depierreux, C. Goyon, G. Loisel, V. Yahia, and J. Rafelski, Nat. Commun. 4, 2506 (2013)

3. A. Picciotto, D. Margarone, A. Velyhan, et al. (Collab.), Phys. Rev. 4, 031030 (2014).

4. C. Baccou, S. Depierreux, V. Yahia, C. Neuville, C. Goyon, R. De Angelis, F. Consoli, J.E. Ducret, G. Boutoux, G. Loisel, J. Rafelski and C. Labaune, Laser Part. Beams 33, 117 (2015)

5. N. Rostoker, M. Binderbauer, and H. Monkhorst, Science 278, 1419 (1997).

6. S. Son and N. J. Fisch, Phys. Lett. A 329, 76 (2004).

7. S. Eliezer and J. M. Martinez-Val, Laser Part. Beams 16, 581 (1998).

8. H. Hora, G. H. Miley, M. Ghoranneviss, B. Malekynia, and N. Azizi, Opt. Commun. 283, 4124 (2009).

9. S.Yu. Gus'kov, Ph. A. Korneev, JETP Letters 104, 1 (2016)

10. N. G. Basov, S. Yu. Gus'kov, and L. P. Feoktistov, J. Sov. Laser Res. 13, 396 (1992).

11. P. A. Norreys, R. Allott, R. J. Clarke, J. Collier, D. Neely, and S. J. Rose, Phys. Plasmas 7, 3721 (2000).

12. S. Yu. Gus'kov, Laser Part. Beams 23, 255 (2005).

13. M. Tabak, J. M. Hammer, M. E. Glinsky, W. L. Kruer, S. C. Wilks, J. Woodworth, M. E. Campbell, M. D. Perry, and R. J. Mason, Phys. Plasmas 1, 1626 (1994).

14. Ph. Korneev, E. d'Humieres, and V. Tikhonchuk, Phys. Rev. E 91, 043107 (2015).

15. Yu. V. Afanasiev and S. Yu. Gus'kov, Nuclear Fusion by Inertial Confinement (CRC Press, Boca Ration, FL, 1993)

16. V. B. Rozanov, C. P. Verdon, M. Decroisette, S. Yu. Gus'kov, J. D. Lindl, K. Nishihara, and H. Takabe, Energy from Inertial Fusion (IAEA, Vienna, 1995) 